\title{
Catheter tip cultures on open-heart surgery patients: associations with site of catheter and age of patients
}

\author{
R FREEMAN, N HJERSING, AND ANITA BURRIDGE
}

\author{
From the Department of Microbiology, Freman Hospital, Newcastle upon Tyne
}

\begin{abstract}
The results of culture of 668 catheter tips from 422 patients are analysed with relation to the site of the catheter and the age of the patient. Coagulase-negative staphylococci were the most common bacterial isolate, but Gram-negative bacilli were found in the venous lines, femoral artery lines, and peripheral lines. Isolations of Gram-negative bacilli were associated with age, being found mainly in those under 1 year and those over 40 years of age. Typing of coagulase-negative staphylococci revealed that Staphylococcus epidermidis is the only variety found in peripheral lines and in lines inserted after operation for complications (central venous, sub-clavian, and long lines), whereas other types occur (along with Staphylococcus epidermidis) in the other lines (left atrial, arterial, and venous). Use of extra lines for the management of postoperative complications thereby increases the risk of Staphylococcus epidermidis gaining access to the circulation. A subgroup of venous lines used to administer inotropic agents was found to be sterile on culture, and this was the result of the anti-oxidant (sodium metabisulphite) in the inotrope solution acting as antiseptic.
\end{abstract}

The organisms isolated from the tips of intravenous and intra-arterial catheters used in openheart surgery patients have been suspected of being one source from which infection of the intracardiac operation site can occur. ${ }^{1}$ This suspicion is strengthened by the similarities of organism types found in the two situations. It has recently been shown that the types of organisms, particularly the coagulase-negative staphylococci, differ with the length of time that the catheters are in situ. ${ }^{2}$ In this study the findings of catheter tip cultures are related to the age of the patient and the site of the catheter.

\section{Methods}

Seven hundred and sixty-eight catheter tips from 422 patients were examined. The patients were unselected and were simply consecutive. Catheter tips were obtained and cultured as previously described. ${ }^{2}$ Organisms isolated were identified by standard methods, thereafter being categorised as

Address for reprint requests: Dr R Freeman, Department of Microbiology, Freeman Hospital, Freeman Road, High Heaton, Newcastle upon Tyne NE7 7DN. coagulase-negative staphylococci (CNS), diphtheroid (Dip), streptococci (Streps), coliform bacilli (Colifs), and yeasts. The term "coliform bacilli" embraces all Gram negative aerobic bacilli, such as Escherichia coli, Proteus, Klebsiella, Pseudomonas, and so on. In some instances the coagulase-negative staphylococci were further subdivided according to the API-staph ${ }^{2}$ method into $S$ epidermidis, $S$ saprophyticus, $S$ hominis, $S$ haemolyticus, $S$ warneri, $S$ capitis, $S$ cohnii. For the purposes of this study these results are expressed as $S$ epidermidis and "other". All the patients were receiving prophylactic flucloxacillin, starting at the time of operation and extending throughout intensive care, apart from 11 patients known to be allergic to penicillins, in whom lincomycin was substituted.

Finally, because of certain results obtained in the study, it was decided to screen certain drugs for anti-bacterial activity against coagulasenegative staphylococci by the following method. A standard blood agar plate was taken and a central well was cut into the plate using a sterile "cork borer". The organisms (a selection of the isolates of coagulase-negative staphylococci from 
this study) were inoculated onto the plate as radial streaks, spreading outwards from the central well like the spokes of a wheel. The substance to be tested was then placed in the central well and the plate incubated overnight at $37^{\circ} \mathrm{C}$. The next day the plate was inspected for signs of inhibition of the bacterial growth, seen as a zone around the well margins. The substances so screened were dopamine, isoprenaline, adrenaline, and $0.1 \%$ sodium metabisulphite.

\section{NOMENCLATURE OF CATHETERS}

The following terms are used. Left atrial (LAthis line is used to monitor left atrial pressure immediately after operation. Drugs are not administered via this route. Venous line (VL) - this line lies in the great veins, in or near the right atrium. Many drugs and fluids are given by this route. Right atrial (RA)-this is a subgroup of the VL category denoting lines through which only inotropic agents are given. Arterial line (AL) - this line is placed in the femoral artery and used to monitor blood gases. All these catheters are inserted in the operating theatre. Peripheral (PER) this line is inserted percutaneously during the operation but is not advanced centrally. A wide range of infusions and drugs is given by this route. Central venous/subclavian/long line catheters (CVP/ SLL) - these catheters are all inserted postoperatively in the Intensive Care Unit, being restricted to those patients in whom complications have developed in the postoperative phase. Uses include parenteral nutrition.

\section{Results}

Table 1 shows the results of culture of the LA lines. Organisms were found in $9.9 \%$ and were almost entirely coagulase-negative staphylococci (CNS). Although numbers were small in the lower age groups there was a tendency for LA tips in the younger patients to be sterile.

As table 2 shows the VL tips yielded organisms in $19 \%$ of cases, the majority of the organisms

Table 1 Isolations from left atrial catheter tips, related to organism type and patient's age

\begin{tabular}{|c|c|c|c|c|c|c|c|}
\hline Age group (yr) & No of catheters & No positive & CNS & Dip & Streps & Colifs & Yeasts \\
\hline $0-1$ & 7 & 0 & - & - & - & - & - \\
\hline $1-5$ & 6 & 0 & - & - & - & - & - \\
\hline $5-10$ & 2 & 0 & $=$ & - & - & - & - \\
\hline & 4 & 0 & - & - & - & - & - \\
\hline $20-30$ & 2 & - & - & - & - & - & - \\
\hline $30-40$ & 10 & 1 & - & 1 & - & - & - \\
\hline $40-50$ & 41 & 5 & 4 & - & 1 & - & - \\
\hline $50-60$ & 65 & 8 & 7 & - & - & 1 & - \\
\hline$>60$ & 24 & 2 & 2 & - & - & - & - \\
\hline Total & 161 & $16(9 \cdot 9 \%)$ & $13(8 \%)$ & $1(0 \cdot 6 \%)$ & $1(0 \cdot 6 \%)$ & $1(0.6 \%)$ & - \\
\hline
\end{tabular}

Table 2 Isolations from venous line catheter tips, related to organism type and patient's age (includes $R A$ catheter subgroup)

\begin{tabular}{|c|c|c|c|c|c|c|c|}
\hline Age group (yr) & No of catheters & No positive & CNS & Dip & Streps & Colifs & Yeasts \\
\hline $0-1$ & 16 & 7 & 3 & 1 & 1 & 2 & - \\
\hline $1-5$ & 10 & 3 & 3 & - & - & - & - \\
\hline $5-10$ & 13 & 0 & - & - & - & - & 一 \\
\hline $10-20$ & 13 & 2 & 1 & 1 & - & - & - \\
\hline $20-30$ & 10 & 3 & 1 & 2 & - & - & - \\
\hline $30-40$ & 7 & 1 & 1 & - & - & - & - \\
\hline $40-50$ & 38 & 5 & 4 & - & - & 1 & - \\
\hline $50-60$ & 56 & 10 & 9 & - & -- & 1 & - \\
\hline$>60$ & 25 & 5 & 2 & - & - & 3 & 一 \\
\hline Totals & 188 & $36(19 \%)$ & $24(12.8 \%)$ & $4(2 \cdot 1 \%)$ & $1(0 \cdot 5 \%)$ & $7(3 \cdot 7 \%)$ & - \\
\hline \multicolumn{8}{|c|}{ RA catheter subgroup } \\
\hline $0-1$ & 2 & ) & & & & & \\
\hline $1-5$ & 1 & & & & & & \\
\hline $5-10$ & 3 & & & & & & \\
\hline $10-20$ & 2 & & & & & & \\
\hline $20-30$ & $\mathbf{0}$ & $>0$ & & & & & \\
\hline $30-40$ & 2 & & & & & & \\
\hline $40-50$ & 4 & & & & & & \\
\hline $50-60$ & 9 & & & & & & \\
\hline$>60$ & 4 & $j$ & & & & & \\
\hline Total & 27 & 0 & & & & & \\
\hline
\end{tabular}


Table 3 Isolations from femoral artery catheter tips, related to organism type and patient's age

\begin{tabular}{|c|c|c|c|c|c|c|c|}
\hline Age group (yr) & No of catheters & No positive & CNS & Dip & Streps & Colifs & Yeasts \\
\hline $0-1$ & 21 & 15 & 8 & - & 3 & 3 & 1 \\
\hline $1-5$ & 15 & 6 & 3 & - & 3 & - & - \\
\hline $5-10$ & 21 & 2 & 2 & - & - & - & - \\
\hline $10-20$ & 17 & 1 & 1 & - & - & - & - \\
\hline $20-30$ & 13 & 3 & 3 & - & - & - & - \\
\hline $30-40$ & 24 & 3 & 3 & - & - & - & - \\
\hline $40-50$ & 60 & 9 & 8 & - & - & 1 & - \\
\hline $50-60$ & 103 & 18 & 15 & - & 1 & 3 & - \\
\hline$>60$ & 41 & 6 & 5 & - & - & 1 & - \\
\hline Total & 315 & $63(20 \%)$ & $48(15 \%)$ & - & $7(2 \cdot 2 \%)$ & $8(2 \cdot 5 \%)$ & $1(0.3 \%)$ \\
\hline
\end{tabular}

Table 4 Isolations from central venous/subclavian/long line catheter tips, related to organism type and patient's age

\begin{tabular}{|c|c|c|c|c|c|c|c|}
\hline Age group (yr) & No of catheters & No positive & CNS & Dip & Streps & Colifs & Yeasts \\
\hline $0-1$ & 1 & 1 & 1 & - & - & - & - \\
\hline $1-5$ & 0 & 0 & - & - & - & - & - \\
\hline $5-10$ & 1 & 0 & - & - & 一 & - & - \\
\hline $10-20$ & 4 & 1 & 1 & - & - & - & - \\
\hline $20-30$ & 0 & 0 & - & - & - & - & - \\
\hline $30-40$ & 7 & 3 & 2 & 1 & - & - & - \\
\hline $40-50$ & 10 & 1 & 1 & - & - & - & - \\
\hline $50-60$ & 16 & 9 & 7 & - & - & 1 & 1 \\
\hline$>60$ & 13 & 12 & 11 & - & 1 & - & - \\
\hline Total & 52 & $27(52 \%)$ & $23(44 \%)$ & $1(1.9 \%)$ & $1(1.9 \%)$ & $1(1.9 \%)$ & $1(1.9 \%)$ \\
\hline
\end{tabular}

Table 5 Isolations from peripheral catheter tips, related to organism type and patient's age

\begin{tabular}{|c|c|c|c|c|c|c|c|}
\hline Age group (yr) & No of catheters & No positive & CNS & Dip & Streps & Colifs & Yeasts \\
\hline $0-1$ & 7 & 7 & - & - & 3 & 5 & - \\
\hline $1-5$ & 2 & 1 & 1 & - & - & - & - \\
\hline $5-10$ & 3 & 1 & 1 & - & - & - & - \\
\hline $10-20$ & 1 & 0 & - & - & $=$ & - & - \\
\hline $20-30$ & 0 & 0 & 二 & - & 二 & - & - \\
\hline $30-40$ & 5 & 1 & 1 & - & - & - & - \\
\hline $40-50$ & 7 & 1 & - & - & 1 & - & - \\
\hline $50-60$ & 20 & 4 & 1 & - & - & 3 & - \\
\hline$>60$ & 7 & 5 & 3 & - & 1 & - & 1 \\
\hline Total & 52 & $20(38 \%)$ & $7(13 \%)$ & 0 & $5(9.6 \%)$ & $8(15 \cdot 3 \%)$ & $1(1.9 \%)$ \\
\hline
\end{tabular}

again being CNS, although other organisms were more common in these lines than in the LA lines. However, such organisms as coliforms and streptococci were found only in the very young and the middle-aged to elderly. Of great interest is the finding that those VL lines used purely for inotropic agents (designated RA lines) were uniformly sterile. Femoral artery catheter tips (table 3) yielded organisms in $20 \%$ of instances, CNS being the most common organisms and such other organisms as coliforms and streptococci again being found in the very young and those over 40 years.

Table 4 shows that CVP/SLL tips frequently produced organisms on culture $(52 \%)$, almost all the organisms being CNS.
As table 5 shows peripheral (PER) lines yielded organisms in $38 \%$ of instances, coliforms and CNS being the most common isolates. The coliform isolates were found, as in VL and femoral artery lines, predominantly in the very young. However, these numbers were small.

The results of typing the CNS from the various sites (table 6) shows that all the lines which were inserted into major vessels at the time of operation (LA, VL, AL) yielded a mixture of $S$ epidermidis and the other types, whereas the PER lines and the lines inserted into major vessels postoperatively (CVP/SLL) yielded CNS which were wholly $S$ epidermidis.

Finally, the three inotropic agents (dopamine, adrenaline, and isoprenaline) tested for anti- 
Table 6 Results of typing of coagulase-negative staphylococci from various catheter sites

\begin{tabular}{llcc}
\hline Catheter site & $\begin{array}{l}\text { No of organisms } \\
\text { typed }\end{array}$ & S epidermidis & Other* \\
\hline LA & 10 & 6 & 4 \\
RA & -- & - & -16 \\
FA & 41 & 25 & 9 \\
VL & 19 & 10 & 0 \\
CVP/SLL & 12 & 5 & 0 \\
PER & 5 & & \\
\hline
\end{tabular}

*See text.

staphylococcal activity were all capable of this, but the activity was found to result from the sodium metabisulphite present in all such compounds as an anti-oxidant.

\section{Discussion}

Left atrial catheter tips are the obvious contenders to be the source of postoperative endocarditis because of the juxtaposition of the catheter tip to the newly inserted valve. It is, therefore, disturbing that $9.9 \%$ yield an organism on culture. Previous reports have emphasised the importance of early removal in diminishing this risk, but it remains unacceptably high. The apparent rarity with which LA catheters yield organisms in younger patients is of interest, since it is commonly held that postoperative endocarditis is rarer in young patients undergoing correction of congenital heart defects than aduits undergoing valve surgery. The explanation that the jet lesion of valve disease predisposes the older patient unduly may well be true,$^{3}$ but this study also suggests that the fact that no organism was isolated from 21 LA lines taken from patients under 30 years of age, compared with 16 isolations from 140 LA tips taken from older patients may have relevance in this context. The finding that the subgroup of VL cultures which had only inotropic agents infused were sterile is of considerable interest. Since the isolation rate of VL lines is $19 \%$ overall, it would be expected that some at least of the 27 catheters in this "RA catheter" subgroup would yield organisms. Even allowing for the reduction in handling when only inotropic infusions were used, the isolation rate could be expected to approach that of the LA lines (about 10\%) and, thus, 27 consecutive sterile catheter tips, while possibly a chance result, might imply some protective mechanism. For these reasons the simple experiment described was performed, and it seems that the sodium metabisulphite incorporated into the inotrop: infusions is also acting as an antiseptic.
It is well known that this substance has such properties. ${ }^{4}$ Since it has been administered to numerous cardiac patients over many years without any obvious side-effects the possible exploitation of this observation may be very important. Experiments to assess these possibilities are in progress.

Femoral artery tips might be expected to yield a variety of organisms because of frequent handling and the site in the groin. It might further be expected that these problems would be particularly common in babies because of incontinence, and both these predictions are borne out by the results. Again, the patients over 40 years of age seem to mirror the results found in the very young, albeit to a lesser degree. Much the same picture emerges in the results from the tips of peripheral catheters (table 4). Thus, while CNS form the bulk of the problem in relation to catheter organisms, it seems that two groups of patients, the babies and adults over the age of 40 years, are prone to catheter colonisation with coliform organisms (and, to a lesser extent, with streptococci). It follows from this that the measures taken to reduce such colonisation may need to be different for these at risk groups. While it is common practice to screen babies for streptococci and coliforms in various sites, since they seem particularly prone to develop infections with such organisms, it may prove profitable to apply such measures to patients over 40 years of age as well. An assessment of this type would possibly pave the way for control of this problem.

Finally, the isolations from CVP/SLL tips reveal a very high rate of infection, almost entirely caused by CNS. This may well be because such lines are used on patients with postoperative complications, thus making it likely that such lines are frequently handled, and the nature of the substances infused. For instance, parenteral feeding is associated notoriously with sepsis of the infusion lines.

It is also of great interest that the types of CNS found in the catheters inserted in the operating theatre into great vessels are a mixture of $S$ epidermidis and other types, whereas the CNS found in the peripheral line (inserted percutaneously) and the CVP/SLL lines (also percutaneous) are all $S$ epidermidis. It is reasonable to assume that $S$ epidermidis is introduced into the line from without-that is, it is a skin organism spreading centrally, access being via the line. The nonepidermidis CNS types, however, might possibly arise from other sources, notably mucous surfaces or the gut. It is particularly interesting that of the 
three patients seen in this hospital with postoperative endocarditis caused by CNS, two had infections with $S$ epidermidis and the third with $S$ saprophyticus. The two patients with $S$ epidermidis infections had a prolonged postoperative course and had CVP/SLL lines used for some time. The patient with $S$ saprophyticus infection had no postoperative problems and had no such lines used.

In conclusion, this study demonstrates several points. Firstly, although it has been shown that all catheters carry some risk of colonisation, it appears that the degree and type of risk varies from site to site, and with age. Thus, coliform organisms and streptococci are a feature of the venous, arterial and, to a lesser extent, the peripheral lines, but are found mainly in the very young and those over 40 years of age. Knowing the groups which are prone to such organisms will help in planning a rational strategy for prevention of colonisation with these organisms which are of varieties likely to be involved in early posto-erative septicaemia. Secondly, it has been shown that CNS are found in all sites, but that the likely pathogenesis differs between those catheters inserted into major vessels at operation (venous, arterial, and left atrial lines) and those inserted percutaneously. It is clear that the use of CVP/SLL lines in patients experiencing postoperative complications dramatically increases the risk of $S$ epidermidis colonisation. Finally, the finding that the small amount of sodium metabisulphite present in the inotrope infusions may prevent colonisation of the lines down which these agents are given may point the way to a simple means of preventing such colonisation in other lines at other sites.

\section{References}

1 Freeman R, King R. Analysis of results of catheter tip culture; in open-heart surgery patients. Thorax 1975; 30:26-30.

2 Freeman R, Hjersing N. Studies on the species of coagulase-negative staphylococci isolated from catheter tips from open-heart surgery patients. Thorax 1980; 35:359-62.

3 Yeh J, Anabtawi IN, Cornett VE et al. Bacterial endocarditis following open-heart surgery. $A n n$ Thorac Surg 1967; 3:29-36.

4 Martindale The Extra Pharmacopoea. Twentyseventh edition. London: The Pharmaceutical Press, 1979; 1284-5. 